\title{
30 Years of Sensors' Assembly and Packaging 1988 to 2018
}

\author{
Juergen Wilde* \\ Department of Microsystems Engineering (IMTEK), \\ University of Freiburg, Georges-Koehler-Allee 103, 79110 Freiburg, Germany
}

(Received December 20, 2017; accepted March 19, 2018)

Keywords: inertial sensor, molded interconnect device, molding, assembly, packaging, interconnection

In this paper, a review of the developments in the assembly and packaging of electronic sensors over the last three decades is given. This review is limited to mechanical-electrical microsystems (MEMS) and microelectronics-based sensors and their packaging at the device level. The evolution of the packaging cannot be regarded independently from the development of microelectronic sensors and MEMS. The focus of this review will be inertial sensors, which require stringent shielding of the functional structure from the environment. The concepts to achieve such hermeticity have evolved from the module level to the device level, and then to the wafer level. By these steps, it was possible to move from ceramic or metal packages to lowcost plastic packages. In the future, the hermetic thin-film capping of MEMS is expected. The injection-molded thermoplastic housings were further developed towards multifunctionality, comprising mechanical, fluidic, or optical features and also 3D wiring in so-called molded interconnect devices (MIDs). In parallel, the molding technology was adapted in order to provide chip-scale packaging with cavities for sensors.

\section{Introduction to Packaging of Sensors}

The assembly and packaging technology of sensors can be defined by several hierarchic levels. ${ }^{(1,2)}$ A basic sensing element, the transducer is packaged at the zero level and the first level. This provides a device that can be combined with several other components on a wiring substrate. In this way, a module or a printed-circuit-board-based assembly is formed. Together with the interconnections and the housing, this provides the second-level packaging (Fig. 1). In this example, three commercially available sensors for the magnetic field are combined in an orthogonal configuration. In this way, a three-axis magnetic field sensor is generated from microelectronic devices, which had previously been restricted to uniaxial measurements of the magnetic field perpendicular to the chip surface. This allowed the packaging to contribute to functionality as precision is provided by the self-alignment on the substrate. Of course, a sensor also needs a protective housing, which contains the sensor element or module and which provides physical interfaces to the total system.

*Corresponding author: e-mail: juergen.wilde@imtek.uni-freiburg.de https://dx.doi.org/10.18494/SAM.2018.1859 


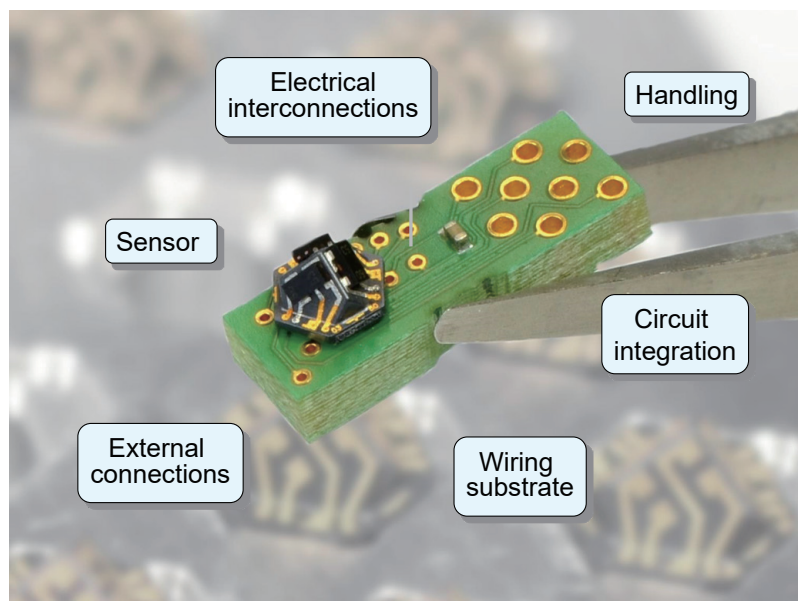

Fig. 1. (Color online) Integration levels and functions of assembly and packaging technology on the example of a molded interconnect device. Image: Hahn-Schickard, project “Adima-3D”.

The contributions of assembly and packaging to the realization of sensors are as follows:

- to form integration and wiring platforms on several levels,

- to mount and fix components on these,

- to provide internal and external electrical connections, as well as

- to make handling and protection possible (Fig. 1).

In these ways, assembly and packaging significantly affect performance, accuracy, reliability, and costs. These factors will be discussed in more detail in this paper.

\section{Developments in Packaging of Inertial Sensors}

Acceleration sensors and gyroscopes are excellent examples to show the developments in the field of sensor packaging over the last 30 years. ${ }^{(3,4)}$ In the 1980 s, both passive and active safety features were increasingly introduced into automobiles. For passenger protection, sensors are needed, which detect high g-forces in the case of a crash. On the basis of this information, protective measures such as the firing of airbags or the tightening of safety belts will be triggered. In order to detect sliding or skidding and for the electronic stability control of a car, gyroscopes were also introduced in addition to accelerometers.

Technically, the sensing principles are based on the effect of linear or angular accelerations on movable masses. Typically, a deflection or internal stress is generated. Capacitive or piezoresistive transducers convert the respective mechanical property into an electrical one using inertial forces such as the Coriolis force. In the transducer, resistances, capacitances, or resonance frequencies are changed in an unequivocal way. An indispensable prerequisite for the proper operation of transducers is that the mechanical structure can move freely, without any contact with other materials except their supports. Otherwise, undesired constraints would lead to frequency shifts and signal damping, which lead to inferior sensing quality. With respect to the high quality factors of resonant sensors, it is desirable that tuning forks, comb 
structures or interdigital structures, as shown in Figs. 2 and 3, are operated under constant or reduced pressures down to the vacuum levels. Therefore, a sealed cavity around the mechanical transducer structure, into which the transducing element is mounted, is required. ${ }^{(5)}$

In the 1980s, the described sensors were no longer "dumb" and passive devices. In order to provide them with a high functionality, a microelectronic circuit was added, which provided instrumentation, drivers, and control circuits as well as A/D and D/A converters, signal processors, and interfaces (Fig. 2). This functionality was achieved in different ways.

The earliest approach was a separate circuit, which was built up by the hybrid integration of a microelectronic integrated circuit (IC), discrete devices, and bare chips on thick-film substrates. In that period, the sensing element was not a MEMS, but it was typically produced by precision machining techniques from metal or ceramic parts. The example shown in Fig. 2 is an acceleration sensor. Up to the 1990s, it comprised a transducer fork made of a ceramic material and had previously been formed from metal. The transducer fork was combined with an actuator that converted the acceleration to an electrical signal. An application-specific integrated circuit (ASIC) drove the transducer and provided instrumentation as well as digital signal processing. Additionally, some passive components such as capacitors and resistors were indispensable. The carrier for this circuit was a hybrid wiring substrate, which was produced using thick-film technology. The base consists of a thin alumina plate on which conductor patterns of noble metals such as AgPd, Au, or AuPt alloys were printed as pastes and then fired at about $850{ }^{\circ} \mathrm{C}$. With thick-film technology, metal- and glass-based multilayers and even integrated resistors could be produced.

The assembly technology for all components was either soldering or adhesive bonding with silver-filled epoxies. The standard interconnection technology from chip to substrate and from substrate to the I/Os was wire bonding, usually $25 \mu \mathrm{m}$ gold wires. For the above-mentioned functionality reasons and also in order to achieve high reliability, the complete hybrid circuit was mounted in a metal package, which was hermetically sealed. The I/O leads were insulated with fused glass pellets.

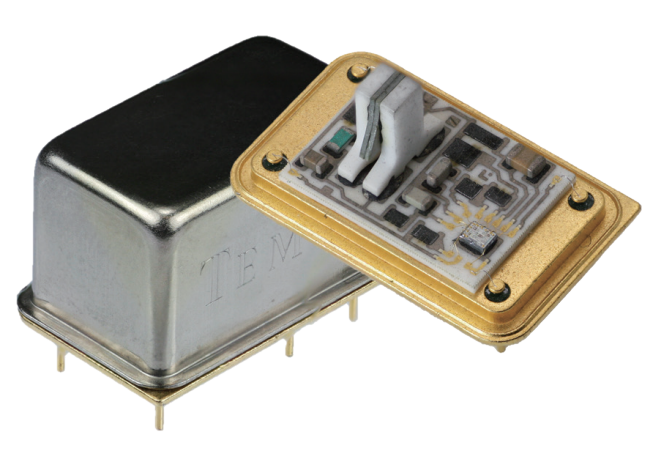

Fig. 2. (Color online) Acceleration sensor assembled from a ceramic transducer, a thick film substrate, an ASIC, and a hermetic metal housing (1990s). Image: IMTEK.

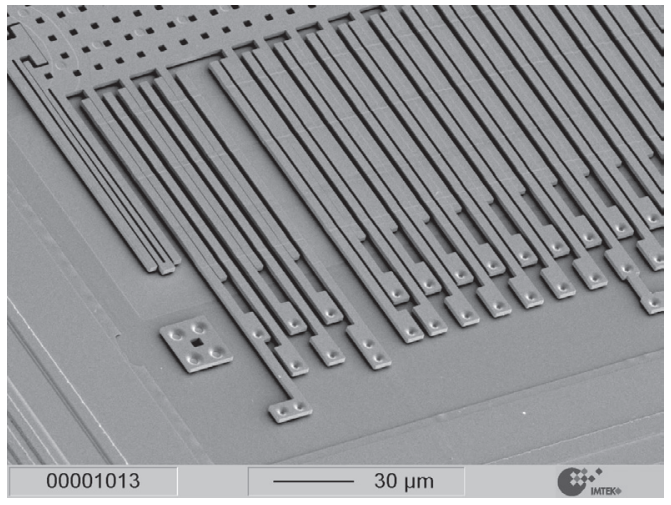

Fig. 3. Surface micromachined transducer structure from a MEMS acceleration sensor, for crash detection (2000s). Image: IMTEK. 
The definition of a hermetic package according to the MIL-STD 883 is that it has a leak rate below approximately $10^{-10} \mathrm{~Pa} \cdot \mathrm{m}^{3} / \mathrm{s}$, as measured with the He leak test, procedure 1014.10. As a second condition, the moisture content must be lower than $3000 \mathrm{ppm}$ by volume. This is equivalent to a dew point of $-10{ }^{\circ} \mathrm{C}$ and is verified with a dew point test or with a mass spectrometer, in accordance with procedure 1018.2. The typical material used for such metal packages was Kovar, a NiCoFe-alloy. Electrical feed-throughs were accomplished using glassinsulated leads (Fig. 2), that have been glass-soldered into openings of the sheet metal. The surfaces to be bonded and in some cases also the interior of the housing were plated with gold in order to improve the wire-bondability and to facilitate water desorption before sealing. For economic reasons, the thick-film hybrids in combination with hermetic metal packaging became obsolete during the 1990s. Still, one of the advantages of the technology is its good commercial availability. Three important developments stimulated the introduction of a new packaging technology that is still predominant in automotive applications.

\subsection{MEMS sensors with monolithic integration}

A significant advancement was the capability to design and fabricate highly complex micromechanical transducers with the surface micromachining (SMM) technology (Fig. 3). This allows the fabrication of acceleration sensors and gyroscopes that fulfill the accuracy requirements of both safety and navigation applications. The relevant properties comprise fast measurement of high accelerations, high resolution of angular rates, and low angular drift. These devices are typically based on capacitive structures of very small capacitance. For highprecision operation, it is necessary that all parasitic capacitances of the assembly and packaging are strictly limited and controlled.

One of the design strategies to accomplish this is the minimization of interconnection lengths to and from the instrumentation circuit. For that purpose, the monolithic integration of the MEMS and $\mathrm{IC}^{(6-8)}$ is optimal. The sensor shown in Fig. 3 provides a complete measurement system on a single monolithic IC for accelerations up to $50 \mathrm{~g}$. For devices with limited instrumentation and signal processing, this was accomplished by the integration of microelectronics and surface micromachined MEMS structures on one chip. The basic research and development efforts for this monolithic integration were carried out in the 1990s. The challenge was that two completely different technologies, micromachining on one side and CMOS with analog circuitry on the other side, had to be united on a single silicon crystal.

There were two possible solutions for this. Either the MEMS structure had to be generated with CMOS compatible processes first, or the MEMS processing was performed after the microelectronics processing. Such sensors exhibit on-chip elements such as the transducer, A/D and $\mathrm{D} / \mathrm{A}$ converters, instrumentation, signal processors, voltage conditioning, and interfaces. ${ }^{(6-8)}$ The first generations of such sensors were still packaged into hermetic ceramic housings (Fig. 4). The example consists of a ceramic small-outline package (SOP). Here, lead frames were attached to prefabricated ceramic shells by glass soldering. The chips were die-attached into these preassemblies with silver-glass material and then wire bonded to the I/O leads. Finally, the lid was again sealed by glass soldering. The features of this solution are the hermetic 


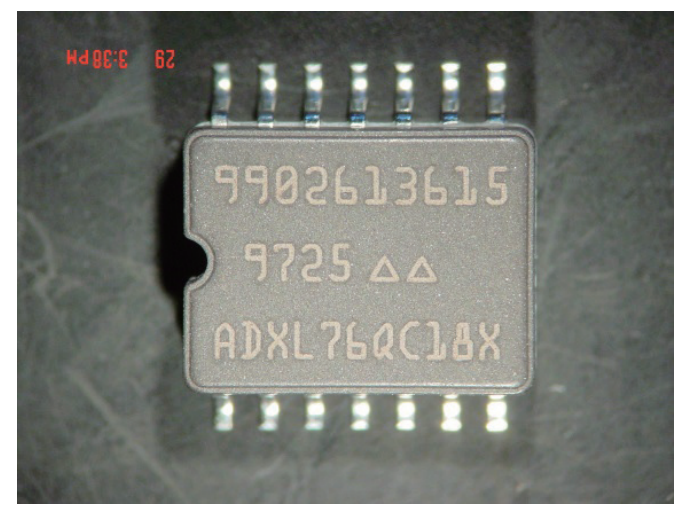

(a)

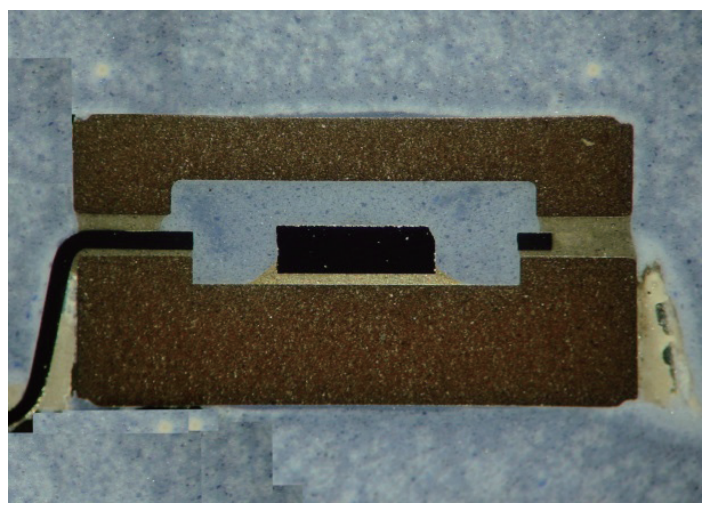

(b)

Fig. 4. (Color online) Hermetic ceramic package for the surface micromachined MEMS acceleration sensor chip from Fig. 3. (2000). (a) Overview of SMD and (b) cross section. Images: IMTEK.

cavity, small size, and the creation of a surface-mountable device (SMD) (Fig. 4). Nevertheless, achieving hermeticity by first-level packaging with ceramic housing can lead to high packaging stresses as a result of the high processing temperatures and will not provide a cost-effective structure.

\subsection{On-chip zero-level hermeticity}

In order to overcome these disadvantages, intensive research led to the second relevant technology, i.e., zero-level packaging. ${ }^{(9,10)}$ Hermetic sealing of the complete assembly was no longer considered as an indispensable requirement for achieving high reliability. Hermeticity could be limited to the area of the device where it was necessary for functional reasons. In this way, a cap must be provided for the MEMS chip, which encloses the MEMS structure in a hermetic cavity. It is necessary that electrical interconnections will be made between the functional structures and the bonding pads on the outside. The cap is in most cases also made of silicon or some borosilicate glass with a very low mismatch between their coefficients of thermal expansion (CTE) against each other. The fusion between the parts can be accomplished by Si-wafer bonding, anodic bonding, AuSn soldering, AuSi eutectic bonding or glass frit bonding (Fig. 5). The lid can either be attached as a single cap or by wafer-to-wafer-mounting. The selection between these alternatives depends on parameters such as chip size, topology, or yield. The example shown is a SMM gyroscope with individually placed caps. While the device already exhibits a monolithically integrated instrumentation circuit, only the MEMS chip is capped (Fig. 5).

With wafer-to-wafer bonding, the top wafer requires openings as some areas of the bottom device wafer must be kept free, in order to provide accessible bonding pads. This can be clearly seen on the cross section of a monolithically integrated acceleration sensor (Fig. 6). The bulkmicromachined (BMM) device consists of three layers: the thick support layer, whose main purpose is to decouple the device from the package mechanically, the center layer, which also 


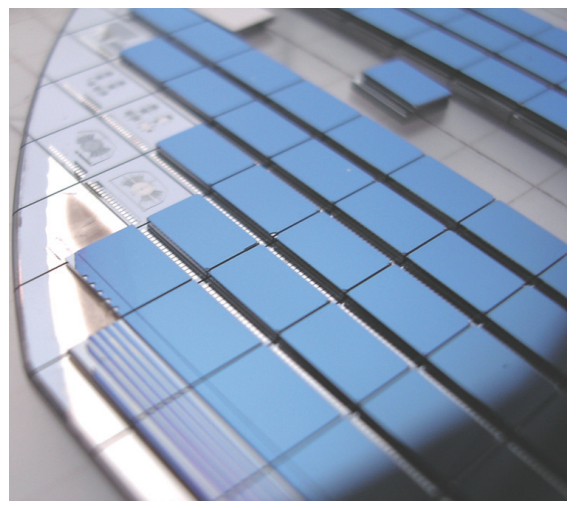

Fig. 5. (Color online) Gyroscope wafer with zero-level on-chip hermeticity, achieved by wafer bonding of a silicon cap on the MEMS wafer (2005). Image: Fraunhofer ISIT.

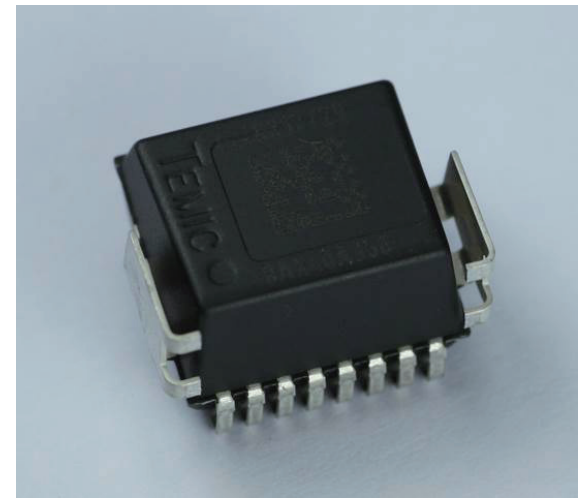

(a)

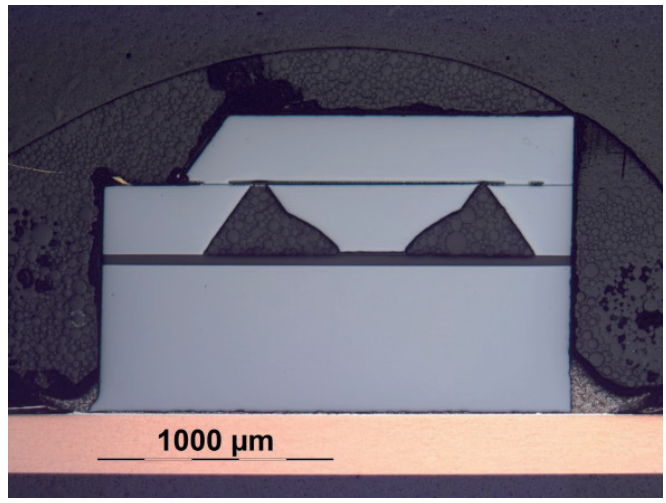

(b)

Fig. 6. (Color online) Zero-level packaging of an acceleration sensor chip with a seismic mass in a cavity and gyroscope wafer with zero-level on-chip hermeticity, achieved by wafer-bonding of a silicon cap on the MEMS wafer (2000). (a) Overview of surface-mountable plastic package and (b) cross section. Images: IMTEK.

contains the functional layer including the interconnections, and the top layer. In this way, a highly efficient technology has been developed, which generates a hermetic cavity with a vacuum atmosphere. Zero-level packaging is one of the most important developments in large series MEMS packaging. It is very efficient to create cavities and to ensure hermeticity. It also contributes to the functional structures of the devices. However, it does not provide a full firstlevel packaging solution. In most mass applications, the user of such components prefers SMD that can further be integrated on printed wiring boards with standard soldering technologies.

\subsection{Plastic packaging}

With the introduction of zero-level hermetic cavities, economic plastic packaging could be utilized for MEMS packaging. There are two principal technologies that were applied: one is the reactive transfer molding technology and the other is the premolded packaging technology (PMP). The two examples shown of the latter are representative of acceleration sensors with 
smaller chips and of gyroscopes with larger chips (Fig. 7). In the cases shown, the MEMS do not have monolithically integrated circuits, but carry only the MEMS structures on chips, which are capped. Although on-chip integration reduces parasitic elements, in many cases, it is more efficient to produce the IC separately. For such decisions, aspects such as process compatibility, complexity, yield, and area utilization must be taken into account.

Figure 7 shows sensors that combine the described wafer-level protected MEMS and separate ASIC chips for instrumentation and signal processing. Both corresponding chips are mounted together in a premolded package, which is designed as a SMD. This package type is a variation of an early SMD, the SOP. These packages are produced by injection molding from high-performance thermoplastic polymers such as liquid crystal polymer (LCP), polyetherether-ketone (PEEK) or polyphenylene sulfide (PPS). These polymers have high thermal stability, are inherently flame retardant, and are less environmentally hazardous than molding compounds containing flame-retarding additives used previously. PMPs typically consist of a metal lead frame made of copper- or nickel-iron alloys, which is embedded into a thermoplastic package body using an injection molding process. ${ }^{(4)}$ Subsequently, the sensor and IC devices are mounted, bonded, and, in the last step, a lid is attached to cover the cavity.

In the field of integrated circuits, reactive transfer molding ${ }^{(11)}$ is still the dominant technology, for economic reasons. The standard technology involves the full embedding of the chip assembly into a polymer molding compound consisting of an epoxy material with a high silica content. Because of the high packaging stresses, even capped MEMS cannot be embedded without further protective measures. There has been significant progress on the adaptation of molding to MEMS. As a result of the soft precoating of the chips or molding with cavities, reactive transfer molding has become increasingly relevant (Fig. 6). In the meantime, chip-scale package types have become available, which are only $20 \%$ larger than the chip. The quality of these first-level plastic packages alone will not be superior to that of hermetic packages with respect to maximum operating temperature or hermeticity. However, combined with protection concepts on wafer-bonded chips, they can be extremely small and will exhibit significant cost advantages.

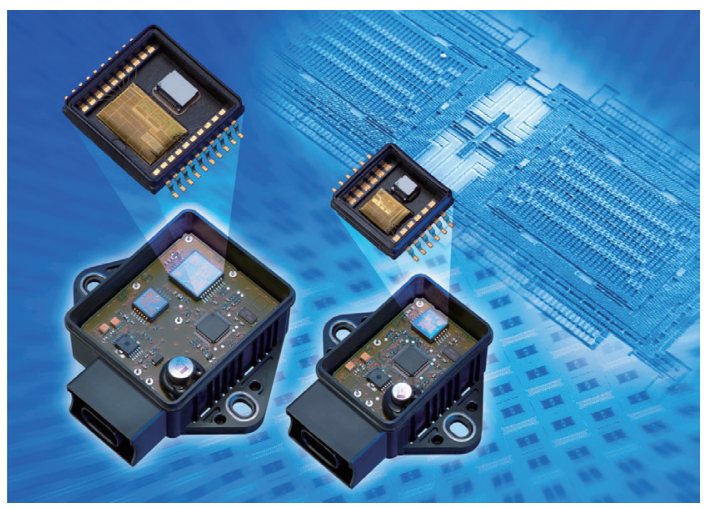

Fig. 7. (Color online) Premolded plastic packaging technology for accelerometers and gyroscopes. Chips are provided with caps for on-chip hermeticity. First-level packaging with premolded surface-mountable plastic package (top), premolded module housings (bottom). Images: BOSCH. 
Besides the first-level packaging of MEMS, premolded technology is also important for the second-level packaging of modules or hybrid circuits (Fig. 7). Such circuits provide functions that are application-specific, as is the case in automotive electronics. In the examples, the sensors were soldered onto the printed wiring boards of electronic control units. The typical features of a module housing are a customer-specific form factor, increased size, integration of an interconnector, and a socket or a flange for attachment in mechanical structures. Secondlevel thermoplastic packages are in most cases application-specific parts. The dominant body materials are poly-butylene-terephthalate (PBT) and polyamides (PA). These materials are composites with up to $40 \%$ glass fibre and inorganic filler.

Particularly in mechatronic applications, a sensor must be attached to a mechanical structure such as an automotive fuel injection system. In order to fit into the system structure mechanically, fixtures (Fig. 7), ducts for gases and liquids, and socketlike electrical interconnectors will have to be integral parts of the housing. The premolded packaging of sensor systems is predominant in many automotive applications. Although these packages are designed to be customer-specific, tooling costs for lead frames and injection molds have limited relevance for the costs per part, because of the large production volumes. Another important factor promoting their use is the high degree of freedom in design. Besides the mechanical elements, ducts for fluids, supports for optical elements, and various kinds of interfaces can also be integrated. As these packages are neither hermetic nor leaktight against moisture penetration, additional silicone potting is required.

\section{Future Developments: Micromachined Sensors with Thin-film Capping}

Despite enormous developments in the field of inertial sensor packaging, future developments can be clearly identified. ${ }^{(10,12)}$ The use of silicon or glass as a cap for the MEMS structure opens several options, such as the creation of several cavities on one chip. This would allow one to produce devices that already have a reference pressure on-chip.

The use of active chips or wafers for capping opens the route to vertical integration and reducing the footprint area of the devices. Nevertheless, advanced processes for vertical interconnection and mounting of flipped or non-flipped ICs will be required. In that case, only wafer-to-wafer techniques appear to be sensible. It is also necessary that the lid wafer has the same pitch as the MEMS wafer, and this can impair the efficiency of the area utilization. Furthermore, the concept is expected to require through-silicon vias, openings for bonding windows in the wafer, and flip-chip interconnections, either as single processes or in combination.

This is the reason why alternatives were evaluated. It is well known that hermeticity can be achieved with metals or with inorganic materials, but not with polymers. Nevertheless, experiences with semiconductor passivation showed that hermeticity is achievable with layers of sub-micrometer thickness, when these are directly attached to the silicon device body. The technique to create a cavity above the MEMS structure is very similar to the basic idea of SMM. The micromechanical structure is embedded into a sacrificial material and subsequently covered with one or more thin layers of very leak-tight materials, such as aluminum, copper, 
nickel, silicon oxide, or silicon nitride. In the next step, the sacrificial polymer is dry-etched from the cavity through an opening, which is sealed again in the last step. As all of the process steps are performed at the wafer level, the process is very efficient and compatible with the SMM technology. Also, no mechanical handling is involved. In this way, inertial sensors with a thin-film cap can be produced very efficiently. ${ }^{(13)}$ An example of an acceleration sensor made with this technology is shown in Fig. 8.

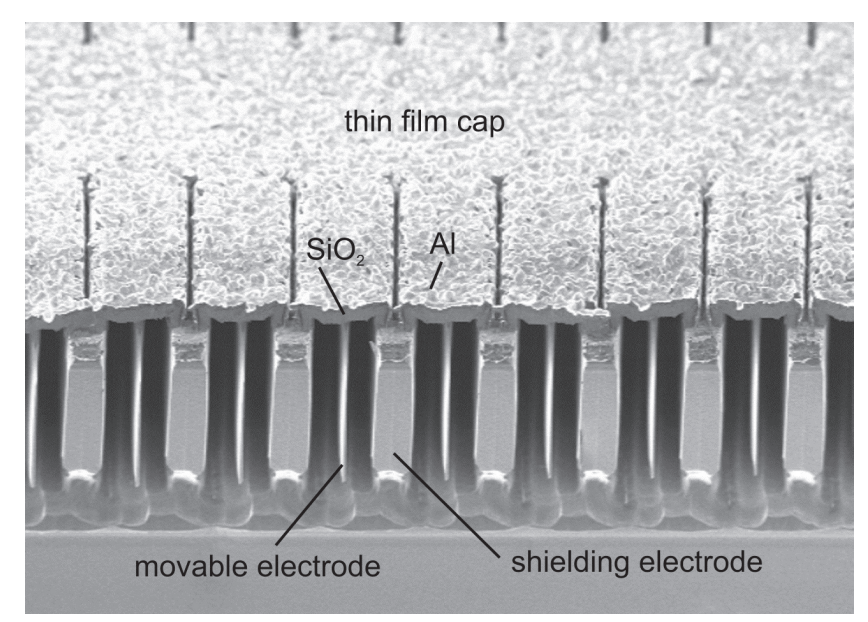

Fig. 8. Cross section of the thin-film-based zero-level capping on a $15 \mathrm{~g}$ acceleration sensor. The chip was made with AIM technology and the thin film consists of $\mathrm{SiO}_{2}$ plus aluminum; a sacrificial layer of $\mathrm{CF}$ polymer is already removed. Image: Fraunhofer ENAS.

Table 1

Comparison of zero- and first-level packaging technologies for sensors and microsystems.

\begin{tabular}{|c|c|c|c|c|c|}
\hline Time period & $1980 \mathrm{~s}$ & $1990 \mathrm{~s}$ & Early 2000s & $\begin{array}{l}\text { Late } 2000 \mathrm{~s} \\
\text { to early } 2010 \mathrm{~s}\end{array}$ & Late 2010 s \\
\hline Transducer & $\begin{array}{l}\text { Precision engineered, } \\
\text { metal or ceramic }\end{array}$ & $\begin{array}{l}\text { Surface micro- } \\
\text { machined SMM }\end{array}$ & $\begin{array}{l}\text { Bulk micro- } \\
\text { machined BMM }\end{array}$ & $\begin{array}{l}\text { Surface micro- } \\
\text { machined SMM }\end{array}$ & $\begin{array}{l}\text { Surface micro- } \\
\text { machined SMM }\end{array}$ \\
\hline $\begin{array}{l}\text { Electronic } \\
\text { circuit }\end{array}$ & ASIC & IC & IC & ASIC & ASIC \\
\hline $\begin{array}{l}\text { Integration } \\
\text { technology }\end{array}$ & $\begin{array}{l}\text { Thick-film } \\
\text { hybrid circuit }\end{array}$ & Monolithic & Monolithic & $\begin{array}{l}\text { Two chips in } \\
\text { one package or } \\
\text { monolithic }\end{array}$ & $\begin{array}{l}\text { Two chips in } \\
\text { one package or } \\
\text { monolithic }\end{array}$ \\
\hline $\begin{array}{l}\text { Zero-level } \\
\text { package }\end{array}$ & None & None & $\begin{array}{l}\text { Hermetic } \\
\text { silicon glass }\end{array}$ & $\begin{array}{l}\text { Hermetic } \\
\text { silicon glass }\end{array}$ & $\begin{array}{l}\text { Hermetic Thin-film } \\
\mathrm{SiO}_{2}, \mathrm{Al}\end{array}$ \\
\hline Package & $\begin{array}{l}\text { Hermetic metal; glass } \\
\text { insulation }\end{array}$ & Hermetic ceramic & Molded plastic & Premolded plastic & $\begin{array}{c}\text { Molded or } \\
\text { premolded plastic }\end{array}$ \\
\hline Device & Acceleration & Acceleration & Acceleration & $\begin{array}{l}\text { Gyroscope } \\
\text { Acceleration }\end{array}$ & $\begin{array}{l}\text { Gyroscope } \\
\text { Acceleration }\end{array}$ \\
\hline Costs & - & ○ & + & + & ++ \\
\hline Performance & - & ○ & ० & ++ & ++ \\
\hline Size & - & + & ++ & $\circ$ to + & ++ \\
\hline Reliability & + & + & + & + & + \\
\hline
\end{tabular}




\section{Conclusions}

In this paper, we demonstrated that there has been great progress in the electronic packaging technology of inertial sensors. The developments are summarized in Table 1. Such devices consist of a mechanical transducer and application-specific instrumentation and data processing circuits. The first of these devices for large-series applications consisted of precisionengineered parts, separate transducers, an ASIC, passive components, a thick-film substrate, and a hermetic housing. Since then, several technological developments have taken place and form the basis for the present state-of-the-art.

For the transducers, micromachined elements became available, first using bulk micromachining, and later, surface micromachining of silicon. These techniques allowed further achievements, such as zero-level packaging, which generates a cavity over the MEMS structure and eliminates the need for hermeticity on the first or second packaging level. In addition, micromachined devices can be integrated with electronic circuits on the same chip. In this way, monolithic sensors can be produced. These developments at the device level were the prerequisites for progress in the packaging. On the first level, this has led to chip-scale surfacemounted devices by encapsulation with plastic materials using reactive transfer molding or by injection molding. The next step towards the fabrication of micromachined sensors with a cavity will probably be thin-film capping of the functional structures of the transducers. This will lead to further progress in integration, miniaturization, and cost reduction.

\section{Acknowledgments}

The author is grateful to his colleagues from Bosch, the Fraunhofer institutes ISIT and ENAS, and the Hahn-Schickard organization.

\section{References}

1 M. L. Kniffin and M. Shah: Int. J. Microcircuits Electron. Packag. 19 (1996) 75.

2 A. P. Malshe, S. B. Singh, W. P. Eaton, C. O’Neal, W. D. Brown, and W. M. Miller: Int. J. Microcircuits Electron. Packag. 22 (1999) 233.

3 N. Barbour and G. Schmidt: IEEE Sensors J. 1 (2001) 332.

4 N. Yazdi, F. Ayazi, and K. Najafi: Proc. IEEE 86 (1998) 1640.

5 O. Brand and H. Baltes: Microsyst. Technol. 7 (2002) 205.

6 L. Spangler and C. J. Kemp: Tech. Dig. 8th Int. Conf. Solid-State Sensors and Actuators (Transducers'95) Stockholm, Sweden (1995) 585.

7 H. Seidel, U. Fritsch, R. Gottinger, and J. Schalk: Tech. Dig. 8th Int. Conf. Solid-State Sensors and Actuators (Transducers'95) Stockholm, Sweden (1995) 597.

8 S. J. Sherman, W. K. Tsang, T. A. Core, R. S. Payne, D. E. Quinn, K. H. Chau, J. A. Farash, and S. K. Baum: Tech. Dig. IEEE Electron Devices Meeting (IEDM'92) (1992) 160.

9 S. T. Cho and F. M. Erdmann: Tech. Dig. Solid-State Sensor and Actuator Workshop, Hilton Head, SC (1998).

10 M. Esashi: J. Micromech. Microeng. 18 (2008) 073001.

11 W. M. Stalnaker, L. J. Spangler, G. S. Fehr, and G. Fujimoto: Proc. 1997 Int. Symp. Microelectronics, Philadelphia, PA (1997) 197.

12 A. C. Fischer, F. Forsberg, M. Lapisa, S. J. Bleiker, G. Stemme, N. Roxhed, and F. Niklaus: Microsyst. Nanoeng. 1 (2015) 15005. https://dx.doi.org/10.1038/micronano.2015.5

13 D. Reuter, A. Bertz, M. Nowack, and T. Gessner: Sens. Actuators, A 145 (2008) 316. 
About the Author

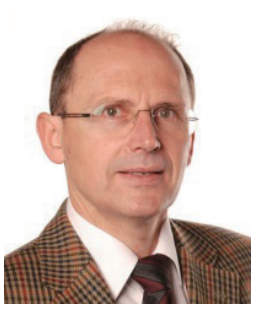

Juergen Wilde received his diploma degree from the University of ErlangenNuremberg, Germany, in 1982 and his doctoral degree from the University of Clausthal, Germany, in 1989. From 1988 to 1999, he was leader of a research group in Daimler Company. Since 1999, he has been a professor at the University of Freiburg's Faculty of Engineering. His research interests lie in the packaging of MEMS, embedded systems, and power electronics for harsh environments. 